\title{
Discretization of Fractional Order Differentiator and Integrator with Different Fractional Orders
}

\author{
Qi Zhang, Baoye Song*, Huadong Zhao, Jiansheng Zhang \\ College of Electrical Engineering and Automation, Shandong University of Science and Technology, Qingdao, China \\ Email: zhangqigogo@163.com, *songbaoye@gmail.com,838294183@qq.com,840428723@qq.com
}

How to cite this paper: Zhang, Q., Song, B.Y., Zhao, H.D. and Zhang, J.S. (2017) Discretization of Fractional Order Differentiator and Integrator with Different Fractional Orders. Intelligent Control and Automation, 8, 75-85.

https://doi.org/10.4236/ica.2017.82006

Received: March 4, 2017

Accepted: May 7, 2017

Published: May 10, 2017

Copyright (c) 2017 by authors and Scientific Research Publishing Inc. This work is licensed under the Creative Commons Attribution International License (CC BY 4.0).

http://creativecommons.org/licenses/by/4.0/

\begin{abstract}
This paper is concerned with the discretization of the fractional-order differentiator and integrator, which is the foundation of the digital realization of fractional order controller. Firstly, the parameterized Al-Alaoui transform is presented as a general generating function with one variable parameter, which can be adjusted to obtain the commonly used generating functions (e.g. Euler operator, Tustin operator and Al-Alaoui operator). However, the following simulation results show that the optimal variable parameters are different for different fractional orders. Then the weighted square integral index about the magtitude and phase is defined as the objective functions to achieve the optimal variable parameter for different fractional orders. Finally, the simulation results demonstrate that there are great differences on the optimal variable parameter for differential and integral operators with different fractional orders, which should be attracting more attentions in the design of digital fractional order controller.
\end{abstract}

\section{Keywords}

Fractional Order Control, Fractional Order Operator, Al-Alaoui Transform, Discretization

\section{Introduction}

Fractional order calculus has a history of more than 300 years, which extends the order of the classical calculus from integer number to arbitrary real number and even complex number. Compared with integral order calculus, the fractional order calculus could describe the dynamic characteristics of the actual system more accurately. Therefore, fractional order control is increasingly becoming one of the most important topics in control theory in recent years [1]. 
The discretization of the fractional-order differentiator and integrator is the foundation of the digital realization of fractional order controller. Generally, there are two methods for the discretization of the fractional-order differentiator and integrator [2], i.e., direct discretization and indirect discretization, while the former is more practical in real applications [3]. Several algorithms have been proposed for the direct discretization method, e.g., the power series expansion (PSE) of the Euler operator, the continued fractional expansion (CFE) of the Tustin operator, etc. [4].

The discretization of the fractional-order differentiator is taken for example. The direct discretization method could be summarized as the following two steps. Firstly, some kind of generating function $\omega\left(z^{-1}\right)$ is used to discretize the differentiator $s$, i.e., $s^{r}=\omega^{r}\left(z^{-1}\right)$, where $r$ is the order of the fractionalorder differentiator and $\omega\left(z^{-1}\right)$ is usually expressed as a function of the complex variable $z$ or the shift operator $z^{-1}$, and then some kind of expansion method is applied to generate the approximate digital filter of the differentiator. For example, Chen and Vinagre propose an IIR (infinite impulse response)-type digital fractional-order differentiator with weighted sum of Simpson integration rule and the trapezoidal integration rule [3]. Al-Alaoui operator and CFE are applied in the discretization of fractional-order operator for better discretization approximation [5]. Zhu and Zou propose an improved recursive algorithm for fractional-order system solution based on PSE and Tustin operator [6]. Miladinovic and his colleagues use genetic algorithm to minimize the deviation in magtitude and phase responses between the original fractional order element and the rationalized discrete time filter in IIR structure [7]. The discretization methods for fractional-order differentiator are compared based on Tustin operator and three different expansion algorithms in [8]. A class of generating function called parameterized Al-Alaoui transform is presented and the simulation analysis indicates that the variable parameter could be adjusted to achieve certain optimal digital filter approximation for the fractional-order operator [9]. However, the variable parameter of the optimal digital filter is different for the fractional-order operator with different orders, this issue has not been intensively discussed in previous papers and will be studied in this paper. The main contributions of this paper are outlined as threefold. (1) The parameterized Al-Alaoui transform is analysed thoroughly to show the effect of the variable parameter. (2) A weighted square integral index about the magtitude and phase is defined as the objective functions to obtain the optimal variable parameter for different fractional orders. (3) Some simulations are implemented to demonstrate the great differences on the optimal variable parameter for differential and integral operators with different fractional orders.

The remainder of this paper is organized as follows. The preliminary of fractional calculus is briefly introduced in Section II. The discretization of fractionalorder operator with different orders is discussed in Section III. Finally, the conclusions are given in the last section. 


\section{Preliminary of Fractional Calculus}

\section{A. Definition of Fractional Calculus}

Fractional order calculus is a natural generalization of the classical integral order calculus, which extends the order of the integration and differentiation to the non-integer or fractional order [10]. Two of the commonly used definitions of the fractional calculus are the Grünwald-Letnikov (GL for short) definition and the Riemann-Liouville (RL for short) definition. The GL definition is defined as

$$
{ }_{a} \mathscr{T}_{t}^{r} f(t)=\lim _{h \rightarrow 0} h^{-r} \sum_{j=0}^{\left[\frac{t-a}{h}\right]}(-1)^{j}\left(\begin{array}{l}
r \\
j
\end{array}\right) f(t-j h),
$$

where $a_{a}{ }^{r}$ is the fractional-order calculus operator, $a$ and $t$ are the limits of the operator respectively, $r$ is the order of the operator and $[\cdot]$ means the integer part. The RL definition is defined as

$$
{ }_{a}{ }_{t}^{r} f(t)=\frac{1}{\Gamma(n-r)} \frac{\mathrm{d}^{n}}{\mathrm{~d} t^{n}} \int_{a}^{t} \frac{f(\tau)}{(t-\tau)^{r-n+1}} \mathrm{~d} \tau,
$$

where, $n-1<r<n$ and $\Gamma(\cdot)$ is the Gamma function. Actually, the aforementioned definitions are equivalent to each other in the real physical systems and engineering applications.

The Laplace transform of fractional order calculus with zero initial conditions for order $r$ is

$$
\mathscr{L}\left\{\mathscr{T}_{t}^{r} f(t)\right\}=s^{r} \mathscr{F}(s),
$$

where $\mathscr{S}\{\cdot\}$ denotes the operation of Laplace transform, $\mathscr{F}(s)$ is the Laplace transform of function $f(t)$ and $s^{r}$ denotes the fractional-order operator [11]. The discretization of fractional-order operator is to design a digital filter to approximate the operator $s^{r}$, where $r$ could be positive or negative for differentiator and integrator, respectively.

\section{B. Parameterized Al-Alaoui Transform}

The discretization of the fractional-order differentiator and integrator is to design a digital filter for the fractional-order operator $s^{r}$. Firstly, a generating function is used to realize the transform of the Laplace operator from the continuous complex frequency domain to the discrete complex frequency domain. According to the definition of $z$ transform, the mapping relationship between the two domains is

$$
z=e^{s T},
$$

where $T$ is the sampling period. Furthermore, the equivalent relation can be formulated

$$
Z=e^{s T}=e^{s((1-\alpha) T+\alpha T)}=\frac{e^{(1-\alpha) T s}}{e^{-\alpha T s}},
$$

where $\alpha \in[0,1]$. Take power series expansion to the numerator and denominator of Equation (5) and neglect the high-order terms, Equation (5) can be 
approximated as

$$
z=\frac{\sum_{n=0}^{\infty}[(1-\alpha) T s]^{n} / n !}{\sum_{k=0}^{\infty}(-\alpha T s)^{k} / k !} \approx \frac{1+(1-\alpha) T s}{1-\alpha T s},
$$

and then the complex variable $s$ can be solved to yield

$$
s=f(z, \alpha)=\frac{1}{T} \frac{z-1}{1+\alpha(z-1)} .
$$

Equation (7) is defined as the $\alpha$ transform from continuous complex frequency domain to the discrete complex frequency domain in [12] and [13]. The analyses presented in [5] and [14] prove that the so-called $\alpha$ transform is actually equivalent to the Al-Alaoui transform [15] [16] with variable parameter $a$ when parameter $\alpha$ is set to $(1+a) / 2$, i.e.,

$$
s=\frac{1}{T} \frac{z-1}{1+\alpha(z-1)}=\frac{2(z-1)}{T[(1-a+(1+a)) z]} .
$$

Specially, three commonly used generating functions, i.e., Tustin transform, Al-Alaoui transform and Euler transform can be formulated when $a$ is set to 0 , $3 / 4$ and 1 , respectively.

A digital integrator with adjustable parameters, which is used in the discretization of fractional-order operator [17], is presented in [18]. The expression of the generating function is

$$
s=\frac{1}{\beta T} \frac{1-z^{-1}}{\gamma+(1-\gamma) z^{-1}},
$$

where $\beta$ and $\gamma$ are gain adjusting parameter and phase adjusting parameter, respectively. The fractional-order operator can be adjusted more accurately with the adjusting parameter according to different real applications. Specially, Equation (9) is equivalent to Equation (7) when parameter $\beta$ is set to 1. Additionally, if the parameter $\alpha$ in Equation (7) is set to $1 /(1+\delta)$, the generating function is formulated

$$
s=\frac{1+\delta}{T} \frac{1-z^{-1}}{1+\delta z^{-1}},
$$

which is used for the discretization of fractional-order operator in [19]. Table 1 shows the relationship of the common generating functions and the variable parameters.

From above analyses, the generating functions in Equations (7), (8), (9) and (10) are actually equivalent to each other when certain relations of the variable parameters are satisfied as shown in Figure 1. We call them parameterized Al-Alaoui transform in [9], a certain expression could be used in certain specific issues.

\section{Power Series Expansion of Generating Function}

Two main methods for the expansion of generating function are continued fractional expansion and power series expansion. Generally, CFE method could 


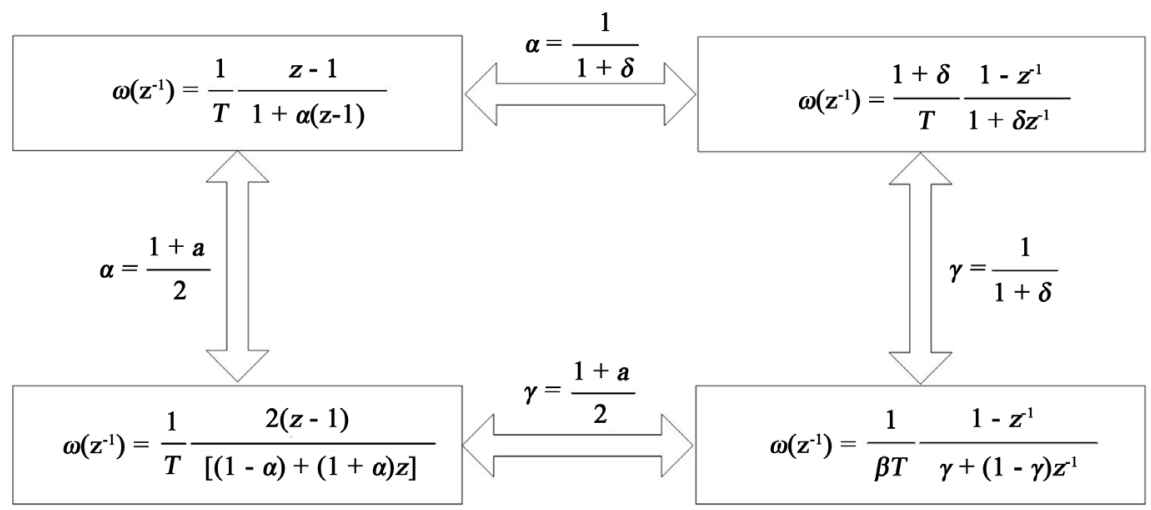

Figure 1. Relations of parameterized Al-Alaoui transform.

Table 1. Relationship of common generating functions and variable parameters.

\begin{tabular}{cccccc}
\hline$\alpha$ & $a$ & $\gamma$ & $\delta$ & $s \rightarrow z$ & Transform \\
\hline 1 & 1 & 1 & 0 & $s^{r} \approx\left(\frac{1-z^{-1}}{T}\right)^{r}$ & Euler \\
$7 / 8$ & $3 / 4$ & $7 / 8$ & $1 / 7$ & $s^{r} \approx\left(\frac{8}{7 T} \frac{1-z^{-1}}{1+z^{-1} / 7}\right)^{r}$ & Al-Alaoui \\
$1 / 2$ & 0 & $1 / 2$ & 1 & $s^{r} \approx\left(\frac{2}{T} \frac{1-z^{-1}}{1+z^{-1}}\right)^{r}$ & Tustin \\
\hline
\end{tabular}

obtain IIR-type digital filter which is easy for the digital filter design, while PSE method could obtain FIR (finite impulse response)-type digital filter and requires less computation cost in comparison with CFE under similar accuracy criterion [4].

In this paper, Equation (10) is used as the generating function, and the power series expansion algorithm presented in [20] is applied to obtain an IIR-type digital filter. The general form of the discretization of fractional-order operator is

$$
\begin{aligned}
\mathscr{Q}^{r}\left(z^{-1}\right) & \approx \operatorname{PSE}\left\{\omega^{r}\left(z^{-1}\right)\right\}_{p, q} \approx \frac{P_{p}\left(z^{-1}\right)}{Q_{q}\left(z^{-1}\right)} \\
& \approx \operatorname{PSE}\left\{\left(\frac{2}{T} \frac{1-z^{-1}}{1+z^{-1}}\right)^{r}\right\}_{p, q} \\
& =\left(\frac{2}{T}\right)^{r} \frac{\operatorname{PSE}\left\{\left(1-z^{-1}\right)^{r}\right\}_{p}}{\operatorname{PSE}\left\{\left(1+z^{-1}\right)^{r}\right\}_{q}} \\
& =\left(\frac{2}{T}\right)^{r} \frac{p_{0}+p_{1} z^{-1}+\cdots+p_{n} z^{-n}}{q_{0}+q_{1} z^{-1}+\cdots+q_{n} z^{-n}}
\end{aligned}
$$

where, $\mathscr{D}^{r}\left(z^{-1}\right)$ is the digital filter of the discrete fractional-order operator, $\operatorname{PSE}\{\cdot\}$ indicates the power series expansion, $P_{p}\left(z^{-1}\right)$ and $Q_{q}\left(z^{-1}\right)$ are the 
numerator and denominator polynomials of the digital filter, and $p$ and $q$ are their orders respectively. Without loss of generality, the approximate orders of the numerator and denominator polynomials are set to $n$ (see [20] for more details).

\section{Discretization of Fractional-Order Operator with Different Orders}

\section{A. Objective Function}

Al-Alaoui transform has better properties in the discretization of fractionalorder operator in comparison with other common generating functions, which have been reported in several papers (see e.g. [4] [7] [21]). In the parameterized Al-Alaoui transform, the parameter $\delta$ could be adjusted to achieve certain optimal digital filter approximation of the fractional-order operator. An objective function is defined in [7], which is to obtain the optimal IIR-type digital filter realization by minimizing the weighted sum of the discrepancies between the responses of the continuous time fractional order filter and its approximate digital filter realization.

However, the papers mentioned above usually consider certain specific fractional order (e.g. fractional order 0.5) and are not concerned with the discretization of fractional-order operator with different orders, which will influence the design of the digital filter. In this paper, we define the following objective functions

$$
\begin{aligned}
& J_{\text {mag }}=\frac{1}{\omega_{b}} \int_{\omega_{l}}^{\omega_{u}}\left\|M_{c}(j \omega)-M_{d}(j \omega)\right\|^{2} \mathrm{~d} \omega \\
& J_{\text {arg }}=\frac{1}{\omega_{b}} \int_{\omega_{l}}^{\omega_{u}}\left\|A_{c}(j \omega)-A_{d}(j \omega)\right\|^{2} \mathrm{~d} \omega \\
& \min J=w_{t} \cdot J_{\text {mag }}+\left(1-w_{t}\right) \cdot J_{\text {arg }}
\end{aligned}
$$

where $M_{c}(j \omega)$ and $M_{d}(j \omega)$ are the magtitude responses of the fractionalorder differ-integrator and its discrete counterpart; $A_{c}(j \omega)$ and $A_{d}(j \omega)$ are the corresponding phase responses; $\omega_{b}$ is the bandwidth betweeen the lower and upper limits $\omega_{l}$ and $\omega_{u}$ (i.e. $\left.\left(\omega_{b}=\omega_{u}-\omega_{l}\right)\right)$ within a chosen frequency band, e.g. $\omega \in\left[10^{-1}, \omega_{N}\right]$ with Nyquist frequency $\omega_{N}$. The normalized $J_{\text {mag }}$ and $J_{\text {arg }}$ depend on not only the parameter $\delta$, but also the different orders $r$. The following simulation analyses are to find the optimal parameter $\delta$ for different fractional orders $r$, which could achieve the minimal objective function $J$ with specific weight $w_{t}$.

\section{B. Simulation Results}

In the simulation, the weight $w_{t}$ is taken as 0.75 without loss of generality. The orders of the power series expansion are taken as 5 for simplicity, the sampling period is taken as 0.001 , and the orders of the fractional-order operator are typically taken as $0.1,0.5,0.9$ and $-0.1,-0.5,-0.9$ for differential and integral operators respectively.

Figures 2-4 are the objective functions for differential operator with fractional order $0.1,0.5$ and 0.9 , where the horizontal coordinate indicates the different 

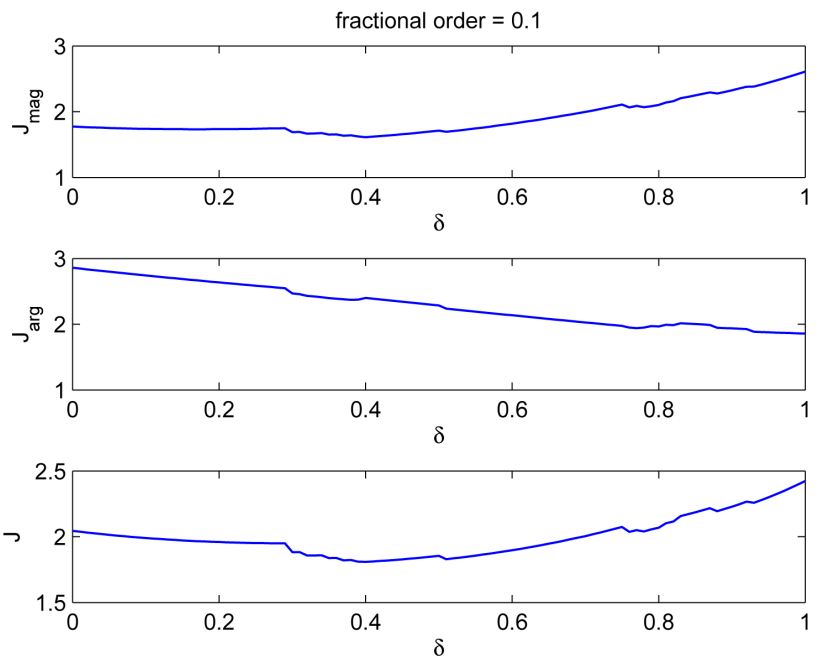

Figure 2. Objective functions for differential operator with fractional order 0.1.
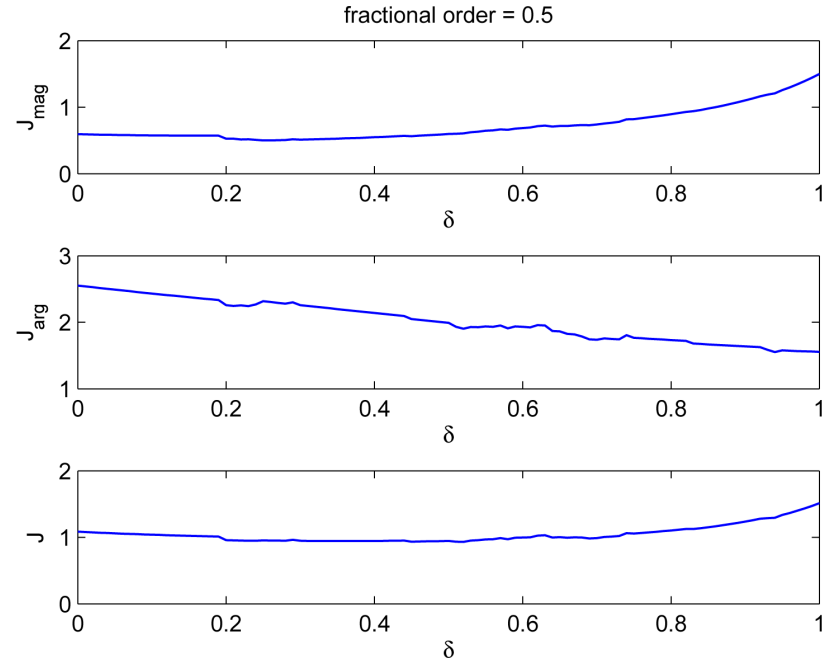

Figure 3. Objective functions for differential operator with fractional order 0.5.
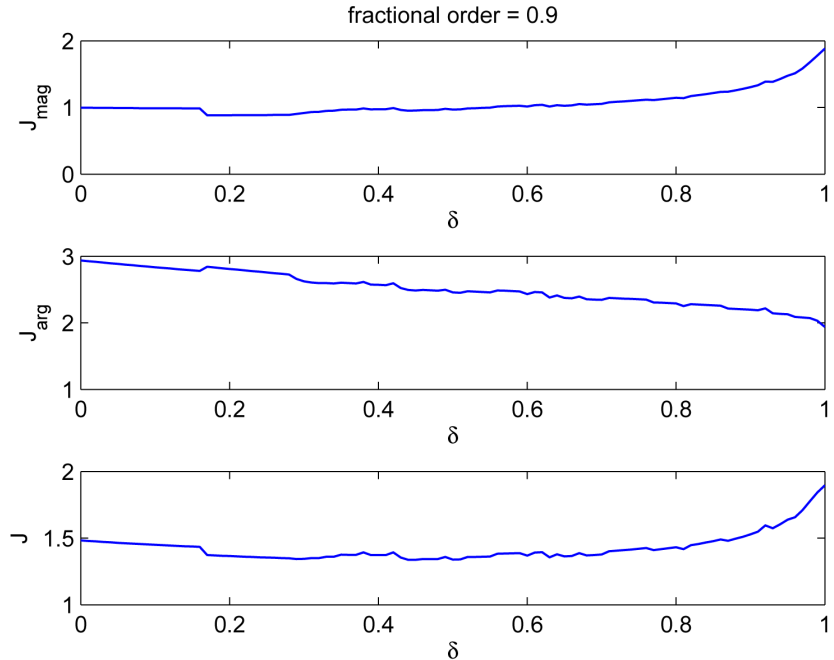

Figure 4. Objective functions for differential operator with fractional order 0.9. 
variable parameter $\delta$ and the longitudinal coordinates indicate the variation of $J_{\text {mag }}, J_{\text {arg }}$ and $J$ respectively. Figures 5-7 are the counterparts of the integral operator. All the figures demonstrate that the variation trends of the objective functions with variable parameter $\delta$ seem consistent with each other, while the optimal variable parameters are totally different for fractional differential and integral operator with different fractional orders under the selected objective functions. Table 2 shows the optimal variable parameters $\delta$ for the specific orders of the differential and integral operators.
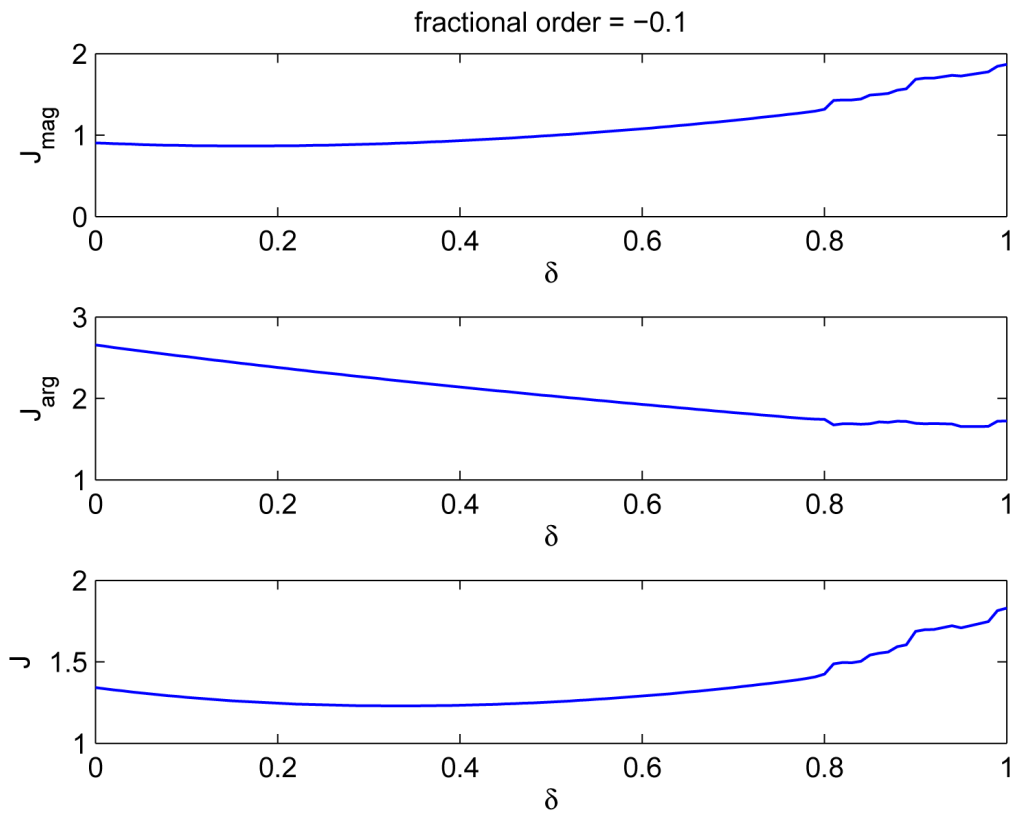

Figure 5. Objective functions for differential operator with fractional order -0.1 .
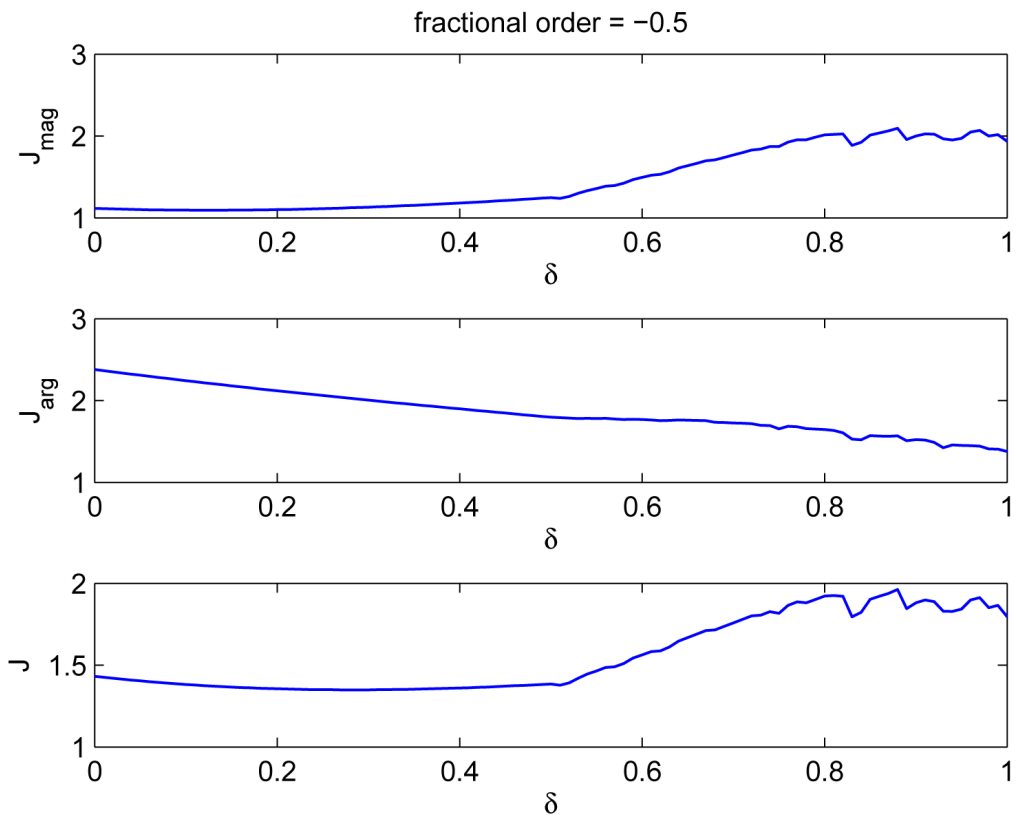

Figure 6. Objective functions for differential operator with fractional order -0.5 . 

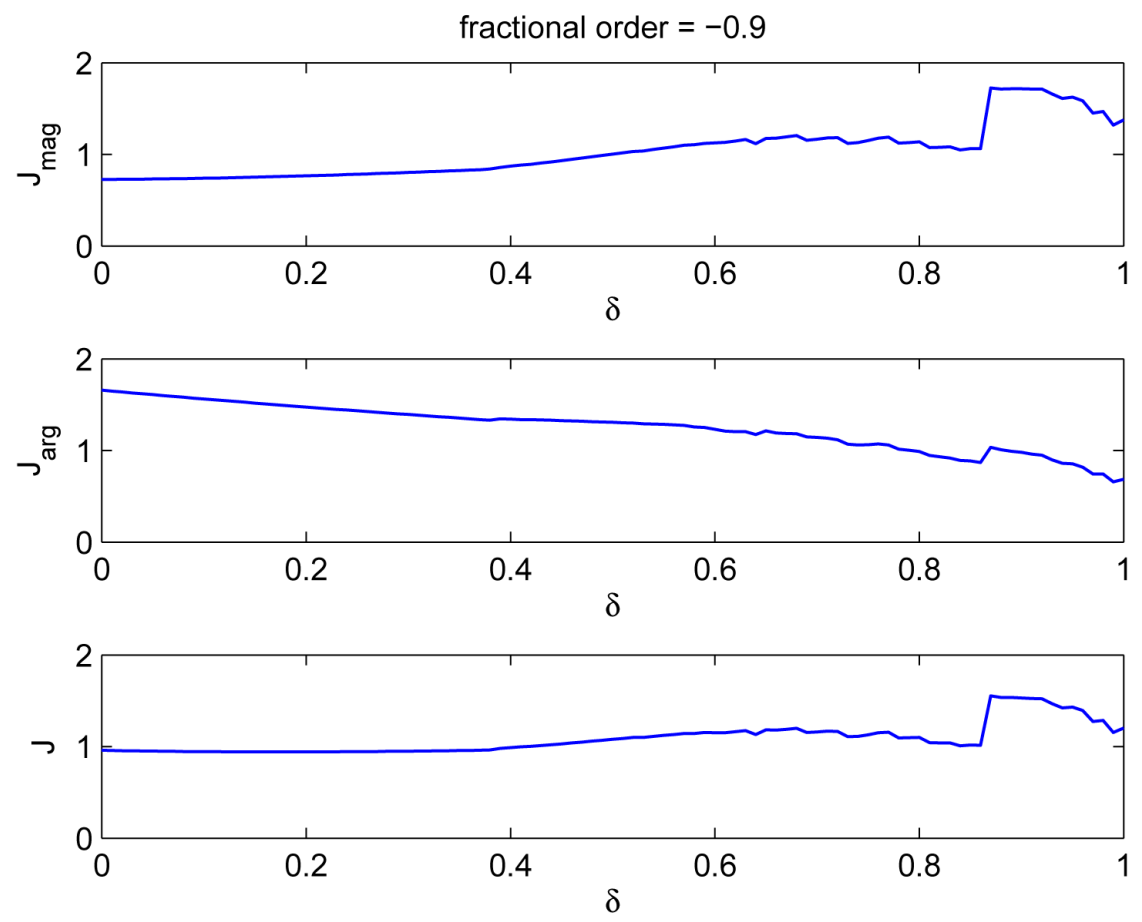

Figure 7. Objective functions for differential operator with fractional order -0.9 .

Table 2. Optimal variable parameter $\delta$.

\begin{tabular}{ccc}
\hline Fractional Order & Optimal $\delta$ for & Optimal $\delta$ for \\
\hline & Differential Operator & Integral Operator \\
0.1 & 0.40 & 0.33 \\
0.5 & 0.52 & 0.28 \\
0.9 & 0.44 & 0.17 \\
\hline
\end{tabular}

\section{Conclusions}

This paper is concerned with the discretization of the fractional-order differentiator and integrator with different fractional orders, which is seldom considered in previous literatures. The parameterized Al-Alaoui transform with one variable parameter is presented as a general generating function, and the objective functions are defined to achieve the optimal variable parameter for the discretization. The simulation results demonstrate that there are great differences on the optimal variable parameters for the discretization of differentiator and integrator with different fractional orders.

However, the weight in the simulation is set as 0.75 without loss of generality, and it is undoubtedly arbitrary to select the proper weight for specific discretization purpose, e.g. select smaller weight for more accurate phase approximation. In the future, we will consider the optimal variable parameter into the practical digital fractional order controller design to acquire the optimal control performances. 


\section{Acknowledgements}

This work is supported by a Project of Shandong Province Higher Educational Science and Technology Program under Grant J14LN34.

\section{References}

[1] Chen, Y., Petráš, I. and Xue, D. (2009) Fractional Order Control-A Tutorial. Proc. American Control Conference, St. Louis, MO, 10-12 June 2009, 1397-1411. https://doi.org/10.1109/acc.2009.5160719

[2] Chen, Y. and Moore, K. (2002) Discretization Schemes for Fractional-Order Differentiators and Integrators. IEEE Transactions on Circuits and Systems I: Fundamental Theory and Applications, 49, 363-367. https://doi.org/10.1109/81.989172

[3] Chen, Y., Vinagre, B. and Podlubny, I. (2004) Continued Fraction Expansion Approaches to Discretizing Fractional Order Derivatives-An Expository Review. Nonlinear Dynamics, 38, 155-170. https://doi.org/10.1007/s11071-004-3752-x

[4] Song, B., Xu, L. and Lu, X. (2014) A Comparative Study on Tustin Rule Based Discretization Methods for Fractional Order Differentiator. Proceedings of International Conference on Information Science and Technology, Shenzhen, 26-28 April 2014, 515-518.

[5] Al-Alaoui, M.A. (2006) Al-Alaoui Operator and the $\alpha$-Approximation for Discretization of Analogue Systems. Facta Universitatis, Ser.: Elec. and Enger., 19, 143-146.

[6] Zhu, C. and Zou, Y. (2009) Improved Recursive Algorithm for Fractional-Order System Solution based on PSE and Tustin Transform. Systems Engineering and Electronics, 31, 2736-2741.

[7] Das, S., Majumder, B., Pakhira, A., Pan, I., Das, S. and Gupta, A. (2012) Optimizing Continued Fraction Expansion Based IIR Realization of Fractional Order DifferIntegrators with Genetic Algorithm. 2011 International Conference on Process Automation, Control and Computing, Coimbatore, 20-22 July 2011, 1-6.

[8] Song, B., Xu, L. and Lu, X. (2015) Discrete Approximation of Fractional-Order Differentiator Based on Tustin Transform. Science Technology and Engineering, 15, 92-95.

[9] Song, B., Xu, L., Lu, X. and Wang, H. (2015) Discretization Scheme for FractionalOrder Differentiators and Integrators Based on Parameterized Al-Alaoui Transform. Proceedings of 34th Chinese Control Conference, Hangzhou, 28-30 July 2015, 6525-6529.

[10] Chen, Y., Vinagre, B., Xue, D. and Feliu, V. (2010) Fractional-Order Systems and Controls-Fundamentals and Applications. Springer, London.

[11] Vinagre, B., Podlubny, I., Hernandez, A. and Feliu, V. (2000) Some Approximations of Fractional Order Operators used in Control Theory and Applications. Fractional Calculus and Applied Analysis, 3, 231-248.

[12] Sekara, T.B. and Stojic, M.R. (2005) Application of the $\alpha$-Approximation for Discretization of Analogue Systems. Facta Universitatis, Ser.: Elec. and Energ., 18, 571586.

[13] Sekara, T.B. (2006) New Transformation Polynomials for Discretization of Analogue Systems. Electrical Engineering, 89, 137-147. https://doi.org/10.1007/s00202-005-0322-2

[14] Al-Alaoui, M.A. (2008) Al-Alaoui Operator and the New Transformation Polynomials for Discretization of Analogue Systems. Electrical Engineering, 90, 455-467. https://doi.org/10.1007/s00202-007-0092-0 
[15] Al-Alaoui, M.A. (1993) Novel Digital Integrator and Differentiator. Electronics Letters, 29, 376-378. https://doi.org/10.1049/el:19930253

[16] Al-Alaoui, M.A. (1997) Filling the Gap between the Bilinear and the Backward Difference Transforms: An Interactive Design Approach. International Journal of Electrical Engineering Education, 34, 331-337. https://doi.org/10.1177/002072099703400405

[17] Barbosa, R.S., Machado, T. and Silva, M.F. (2006) Time Domain Design of Fractional Differintegrators Using Least-Squares. Signal Processing, 86, 2567-2581.

[18] Smith, J.M. (1987) Mathematical Modeling and Digital Simulation for Engineers and Scientist. 2nd Edition, Wiley, New York.

[19] Caponetto, R., Dongola, G. and Fortuna, L. (2010) Fractional Order Systems: Modeling and Control Applications. World Scientific Publishing, Singapore.

[20] Petras, I. (2012) A New Discrete Approximation of the Fractional-Order Operator. 13th International Carpathian Control Conference (ICCC), High Tatras, 28-31 May 2012, 547-551. https://doi.org/10.1109/carpathiancc.2012.6228705

[21] Chen, Y. and Vinagre, B. (2003) A New IIR-Type Digital Fractional Order Differentiator. Signal Processing, 83, 2359-2365.

\section{Scientific Research Publishing}

\section{Submit or recommend next manuscript to SCIRP and we will provide best} service for you:

Accepting pre-submission inquiries through Email, Facebook, LinkedIn, Twitter, etc. A wide selection of journals (inclusive of 9 subjects, more than 200 journals)

Providing 24-hour high-quality service

User-friendly online submission system

Fair and swift peer-review system

Efficient typesetting and proofreading procedure

Display of the result of downloads and visits, as well as the number of cited articles Maximum dissemination of your research work

Submit your manuscript at: http://papersubmission.scirp.org/

Or contact ica@scirp.org 